\title{
A Photonic Model of the Big Bang
}

\author{
J. G. Lartigue* \\ National University of Mexico, Mexico City, Mexico \\ Email: jmlg5@hotmail.com
}

How to cite this paper: Lartigue, J.G. (2018) A Photonic Model of the Big Bang. Journal of Modern Physics, 9, 2443-2456. https://doi.org/10.4236/jmp.2018.914157

Received: November 14, 2018

Accepted: December 17, 2018

Published: December 20, 2018

Copyright $\odot 2018$ by author and Scientific Research Publishing Inc. This work is licensed under the Creative Commons Attribution International License (CC BY 4.0).

http://creativecommons.org/licenses/by/4.0/

(c) (i) Open Access

\begin{abstract}
There are two main theories about the origin of the Universe that show similitude with the Genesis writings, though in different verses: the Big Bang ${ }^{1}$ and the eternal Universe ${ }^{2}$ (an eventual quantum fluctuation). However, it is possible to partially include the quantum theory in the Big Bang thanks to the nature of photons, to obtain a simple model. It is assumed as the origin of the Universe (space, time, matter and physical laws). A subsequent enormous expansion has been explained by a supposed brief Inflation period, followed up today by a constant adiabatic expansion acceleration. This paper assumes that the Universe is the total Space which contains the Physical Universe covered by an external, empty Space, both expanding at a constant Hubble acceleration $\Gamma_{H}$ [1]. A Big Bang design is intended by a deduction of the energy and number of primeval photons, from the present $\mathrm{CMB}$ value; they would have reacted whether to generate the Physical Universe or to decay till the CMB level. It follows an approach to the Universe expansion work, based on the Hubble field $\left(V_{H}\right)$ as well as on Thermo-dynamics. They are calculated: the time and angular momentum required for the Physical Universe to reach the maximum internal velocity $c$ as well as, simultaneously, a $c$ tangential velocity. The Universe luminosity at different periods and the adequate expressions of parameters $(\Omega, q, k)$ are revised. It is proposed a modification in the equation of the $H(t)$ parameter and the $H_{o}$ value. The operator of convective derivative is applied to obtain an equation of continuity for the photonic energy; an adiabatic Jacobian gives similar results. This essay differs from others based on black box radiation, since the Universe has no walls and the photons energy decays continuously.
\end{abstract}

\section{Keywords}

Hubble Parameters, Space Acceleration, Photonic Equation of Continuity, Universe Angular Momentum

\footnotetext{
${ }^{\star}$ Retired Professor.

${ }^{1}$ Genesis, Chapter 1, Verse 3: And God said "let there be light" and there was light.

${ }^{2}$ Genesis, Chapter 1, Verse 2: "The earth was without form and void... and the spirit of God was hovering over the face of waters".
} 


\section{Introduction}

\subsection{Previous Models}

Reference [2] describes the extensive research developed to determine the CRB variations, which may suggest a spectral distribution of the Big Bang original photons. Reference [3] is a complete text on Statistical Physics though its chapter on photon gas is mainly oriented to black body and cavities radiations. A previous classical book [4] mentions the Wien constant, necessary to determine the maximum energy density, though it is exemplified only by solar radiation. Reference [5] assumes that the photon gas is a carrier for electromagnetic waves in a very complex model. However, it was not found a Big Bang thermo-dynamical model that would, probably, exist.

\subsection{The Selected Universe Model}

The Einstein gravitational equation, including the cosmological constant $\Lambda$, was modified by De Sitter for an empty Universe [6]. In reference [7] the $\Lambda g_{\mu v}$ tensor was substituted by a Hubble tensor (whose scalar equals $3 \mathrm{H}^{2}$ ); it implies a Hubble positive potential field $V(r)$ and the expansion acceleration $\Gamma_{\mathrm{H}}$. The photonic model has been selected because the Planck temperature represents an initial energy about $10^{22}(\mathrm{Mev})$, which overpasses the binding energy of any mass, from neutrino to Higgs particle. For simplicity it is initially assumed as a monoenergetic photonic source, which could later be modified as function of a probable Planck Length indetermination.

\subsection{The Light Speed in Dense Matter}

A doubt could be elicited due to the use of the $\mathrm{c}$ value in Planck parameters because, at that time, the linear speed of initial photons could not be higher than that of the space expansion. However, such an obstacle may have overcome by the results of a research on opaque bodies [8] which concluded that the total trajectory of light through a big number of collisions per second corresponds to $c$. That would confirm the light velocity as a true constant in the Physical Universe, provided that the internal mean free path in the mass would be wide enough to admit a wavelength displacement; obviously, the $c$ value had been confirmed in vacuum.

\section{Some Universe Parameters}

\subsection{Previously Published Parameters}

The maximum velocity of matter into the physical Universe is $c$ (Figure 1); the present time is $t_{o}=14 \mathrm{~Gy}$. It has been assumed that the Big Bang duration was $t_{b e}$ $=10^{12}(\mathrm{~s})$ [9] or $3.2 \times 10^{4}(\mathrm{y})$. Besides, the maximum velocity of matter into the physical Universe has been limited to $c$, which was freely manifest at the Universe expansion time $t_{c}=1.1 \times 10^{17}(\mathrm{~s})$; the corresponding Universe scale factor $R_{c}$ (Universe radius $\boldsymbol{r}_{c}$ ), where velocity expansion $\mathrm{c}$ freely occurred, was $\boldsymbol{r}_{c}=1.7$ $\times 10^{27}(\mathrm{~cm})$. Otherwise, it has been shown [1] that the space acceleration 


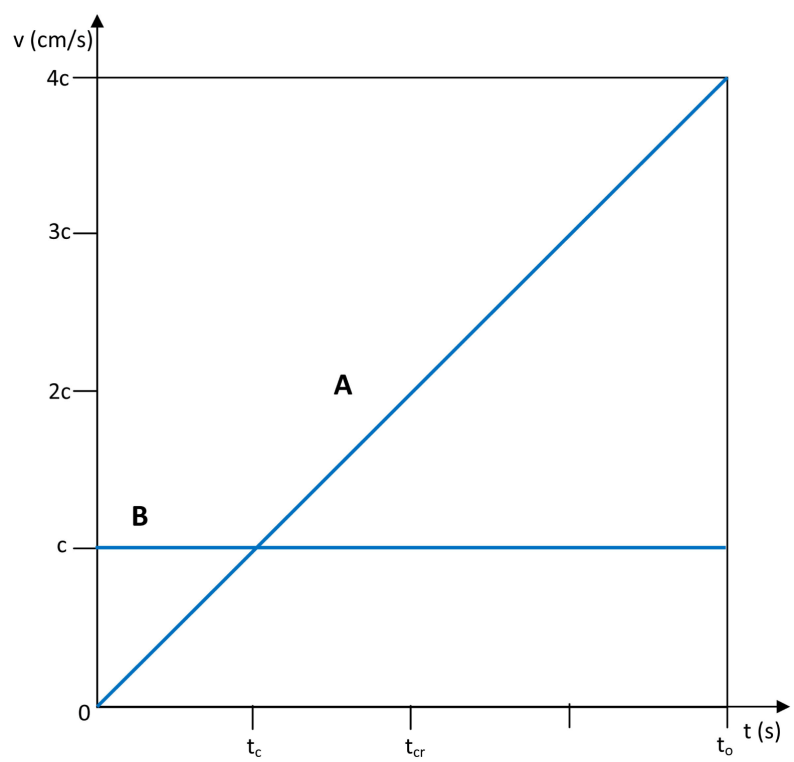

Figure 1. Universe expansion velocity as a time function. A: expansion velocity of Space, Equation (1). B: constant velocity of light in the Physical Universe.

$\Gamma_{H}\left(\mathrm{~cm} \cdot \mathrm{s}^{-2}\right)$ is a constant, independent of mass presence. Present time is $t_{o}$. The Space expansion velocity is:

$$
v_{H}=\Gamma_{H} \cdot t
$$

The value of the Hubble parameter has been here corrected to $H_{o}=3.2 \times 10^{-18}$ $\left(\mathrm{s}^{-1}\right)$ assuming a maximum speed of $100(\mathrm{~km} / \mathrm{sMpc})$ in the definition equation. So, the intensity of the Hubble field is $\Gamma_{H}=2.65 \times 10^{-7}\left(\mathrm{~cm} \cdot \mathrm{sec}^{-2}\right)$ (Figure 2).

\subsection{Additional Parameters}

1) The critical condition of the Universe has been defined in two ways: respect to a ratio $\Omega$ [10] of present and critical densities and respect to a net acceleration [11] as:

$$
\begin{gathered}
\Omega=\rho_{o} / \rho_{c r} \\
\Gamma_{N}=\Gamma_{H}-\Gamma_{G} \\
\Gamma_{H}=H^{2} \boldsymbol{r}=\text { constant } \\
\Gamma_{G}=G M / \boldsymbol{r}^{2}
\end{gathered}
$$

Though Equations (4) and (5) may be here applied, Equations (2) and (3) should not because of the following: Equation (2) is not adequate for the De Sitter model since the vacuum density of the external empty space is $\rho_{o}=0$ and, therefore, $\Omega=0$, independently of the density of the physical Universe. A critical point could rather correspond to the $t_{c}$ time, when the expansion velocity of Space and the internal velocity in the Physical Universe diverge; at that time, the gravitational acceleration is $\Gamma_{G} \sim 10^{-2}$ times lower than the space acceleration $\Gamma_{H}$ (Figure 2). Even more, the eventual numerical equality in Equation (3) occurs at a time $t_{c r} \sim 2 t_{c}$, without any physical consequence. 


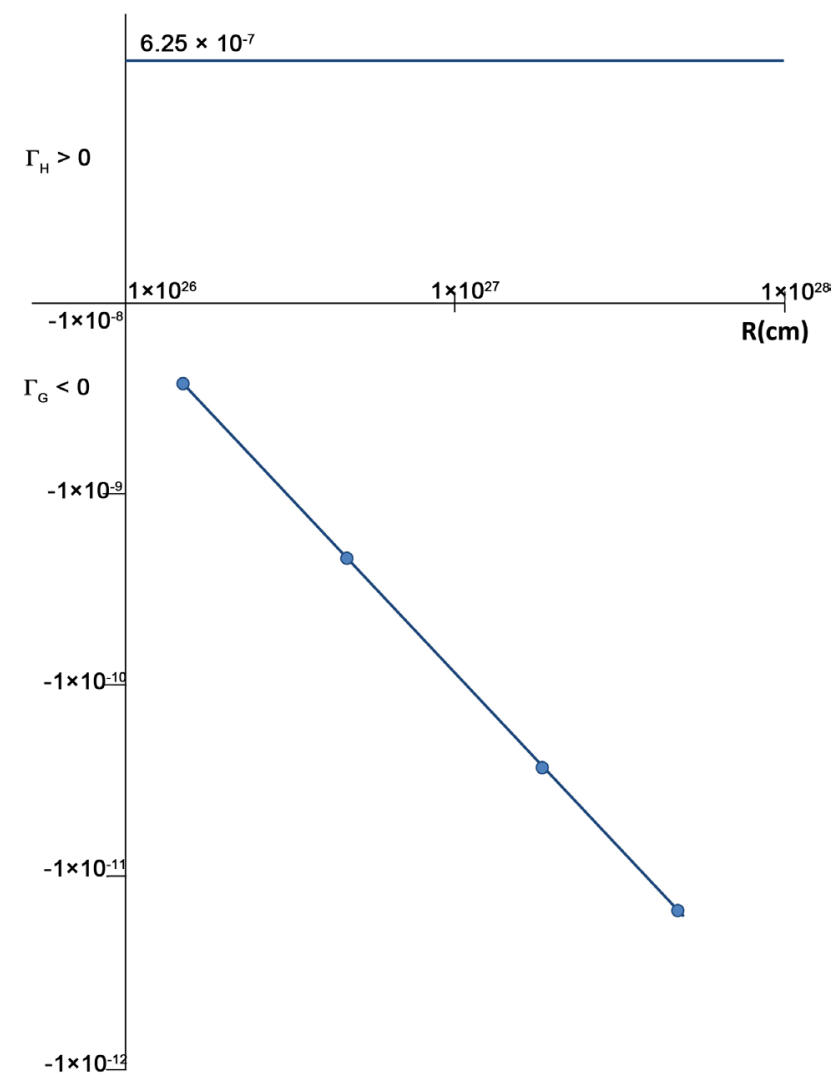

Figure 2. Evolution of the Hubble $\left(\Gamma_{H}\right)$ and gravitational $\left(\Gamma_{G}\right)$ acceleration $\left(\mathrm{cm} \cdot \mathrm{s}^{-2}\right)$ as function of the radial factor $(R)$, Equations (4) and (5).

2) Another important factor is the so-called deceleration factor $q_{u}$ defined [12] by the equation:

$$
q_{u}=-R \ddot{R} / \dot{R}
$$

This expression was considered adequate at the time when the Universe acceleration was assumed negative, accordingly to the second Friedman equation [13]:

$$
\frac{\ddot{R}}{R}=-\frac{4 \Pi G}{3}\left(\rho+\frac{3 p}{c^{2}}\right)
$$

In these two equations $R$ is the scale factor of the Universe, $\dot{R}$ is the expansion velocity and $\ddot{R}$ is the acceleration; as well, $\rho$ is the Universe density and $p$ is its pressure.

To date, at a positive acceleration, the minus sign should be discarded from Equation (6).

3) The curvature of an spherical Universe may be estimated by the classical expression $k=1 /\left(R_{u}\right)^{2}$. If obtained from the first Friedman equation, this gives the following results: at early times, when the Hubble parameter was higher than today, $k$ would be negative, i.e. a hyperbolic geometry; now, at the smaller magnitude of $H_{o}$, the $k$ value is still negative though in a rank of $10^{-40}\left(\mathrm{~cm}^{-2}\right)$ that suggest a flat Universe. Otherwise, the FLRW equation admits 3 possible values 
for $k(+1,0,-1)$ to cover all of curvature possibilities.

4) The Laplacian for the Hubble potential was deduced [1] as $\nabla^{2} V_{H}=0$, since $V_{H}$ is not a function of the mass presence.

\section{The Hubble Parameters}

1) The original parameter was defined by $H$. Hubble as the relation $H=v / c$, later, it has been defined by its own units $\left(\mathrm{s}^{-1}\right)$ in successive concepts: from a universal constant $\left(H_{o}\right)$, to a single reciprocal function of time, $H=(1 / t)$ and even to $H=2 / 3 t$ in the Einstein-De Sitter model.

2) The Hubble time has been defined as $t_{H}=1 / H$. However these equations have been applied for a Universe whose expansion velocity was assumed constant. If the acceleration $\left(\Gamma_{H}\right)$ is the true constant, the Hubble time should be expressed as:

$$
t_{H}=\sqrt{2} / H(t)(\mathrm{s})
$$

3) The Hubble Length, as known nowadays, is defined by the equation:

$$
L_{H}=c / H_{o}(\mathrm{~cm})
$$

Substitution of Equation (8), gives:

$$
L_{H}=c t_{o} / \sqrt{2}(\mathrm{~cm})
$$

Equation (9) is equivalent to that for an inertial frame: $\boldsymbol{r}=\boldsymbol{v} t$, if $\boldsymbol{v}$ had the $\boldsymbol{c}$ value. Equation (10) was obtained for an accelerated frame, $\Gamma_{H}$. So, this equation would be valid for the case when the expansion velocity would be higher than $c$ as: $\boldsymbol{v}_{H}=n \boldsymbol{c}$, where $n \geq 1$, so giving, for the present Hubble length:

$$
L_{H}=n c t_{o} / \sqrt{2}(\mathrm{~cm})
$$

4) The Hubble velocity of Space expansion may be obtained, as a function of time, from the above mentioned Equation (1) if $\boldsymbol{V}_{H}$ is substituted by $\dot{\boldsymbol{R}}$, as $\dot{\boldsymbol{R}}=\Gamma_{H} \cdot t \quad(1 \mathrm{a})\left(\mathrm{cm} \cdot \mathrm{s}^{-1}\right)$.

\section{The $c$ Factor}

It has been assumed [14] that the space expansion velocity could overpass $c$ since the space is not in an inertial frame; rather, it contains all of reference frames. So, the concept of co-moving coordinates would be better applied to Space. From the above results, the $t_{c}$ time has occurred at one fourth of the Universe age, near after the starting of the Physical Universe formation. That means that the light velocity into the Physical Universe has been evidently constant during the last three fourths of the Space age. There is a possible explanation for that: the expansion velocity of Space is not limited to $c$ though matter velocity, into the Physical Universe, is really limited due to the space-time curvature originated by the mass density. However, to obtain a probable image of these subjects, there is no other way but to apply the available means [15] [16]. So it is possible to assume that, in the co-moving coordinates of Space, the expansion 
would be referred to the Big Bang origin $(t=r=m=0)$.

In order to determine the light velocity $c$ as a function of the gravitational potential $\Phi$, at a given distance, Einstein [17] did propose the following equation:

$$
c(\Phi)=c_{o}\left(1+\frac{\Phi}{c_{o}^{2}}\right)
$$

$\boldsymbol{c}_{o}$ is the present, known velocity. However, when applied to a higher potential corresponding to a smaller radius of the Physical Universe, the increase in $c$ results almost negligible; it would confirm the constancy of $c$ since the tiny obtained difference obeys, rather, to the imprecision of data applied in this paper.

Therefore, there are two realities: into the Physical Universe $c$ is a true constant; in the Space, its expansion velocity pulls all matter (including photons) at higher velocities $\boldsymbol{V}>\boldsymbol{c}$. A possible reason for the constancy could be the conservation of the angular momentum in the Physical Universe rotation that, at the $t_{c}$ time, would have had a tangential velocity $c$ which has been necessarily decreasing till the present time. So, if the total angular momentum of the Physical Universe $(\mathcal{L})$ is calculated at the $t_{c}$ time, as:

$$
\mathcal{L}_{c}=M_{U} \cdot r_{c}^{2} \omega
$$

by substitution of the relation $\boldsymbol{V}_{c}=\omega \cdot \boldsymbol{r}_{c}$ it gives:

$$
\mathcal{L}_{c}=M_{U} \cdot \boldsymbol{r}_{c} \times \boldsymbol{v}_{c}
$$

So, the constant value of the Physical Universe angular momentum at the $t_{c}$ time it results: $\mathcal{L}=4.8 \times 10^{94}$ (erg.s); then, the to date tangential velocity of the Physical Universe Limit $\left(R_{p u}\right)$, i.e. $z=11$, would be $\boldsymbol{v}_{p u}^{t}=2 \times 10^{10}(\mathrm{~cm} / \mathrm{s})$.

It has been mentioned that a low gravitational potential has a very few influence in the case of an equilibrium temperature. However, in the non equilibrium temperature and a higher potential case, it seems that such influence would remain small.

\section{The $\gamma$ Factor}

The Space expansion velocity $\left(v_{s}\right)$ has really surpassed the $c$ value after the $t_{c}$ time and S. Hawkings [18] did mention the possibility of an imaginary time. Without a known theory about the empty space kinematics and too far from any gravitational interaction, the Special Theory of Relativity can be applied to obtain some expressions for a frozen and imaginary time, by means of the proper time $(\tau)$ equations:

$$
\tau=t / \gamma
$$

where:

$$
\gamma=1 /\left(1-\frac{v^{2}}{c^{2}}\right)^{0.5}
$$

i.e. 


$$
\tau=t\left(1-v^{2} / c^{2}\right)^{0.5}
$$

So, If $\boldsymbol{v}=\boldsymbol{c}, \gamma=\infty, \tau=0$ (that means a time singularity). If $\boldsymbol{v}>\boldsymbol{c}, \tau$ has an imaginary value, $\tau$. It may be determined the precise value of this imaginary number, by the $\gamma$ factor: If $\boldsymbol{v}>\boldsymbol{c}$, it means that $\boldsymbol{v}=\beta \boldsymbol{c}$, being $\beta$ a real number higher than 1 , so $\beta^{2}>1$; if $w^{2}=1-\beta^{2}, w^{2}<0$ and, from Equation (14),

$$
\tau_{i}=t\left(w^{2}\right)^{0.5}=t \cdot w i
$$

Therefore, when the velocity of some matter reaches $\boldsymbol{V}=\boldsymbol{c}$ and $\tau=0$, that would mean a singularity (a time freezing at a physical Universe radius $r_{c}$ ), reached at one fourth of the present time. After that time $\left(t>t_{c}\right)$, at an expansion velocity $\dot{R}>c$, the proper time of space would become imaginary. Otherwise, as the physical Universe has maintained constant its maximum internal velocity $c$, the proper time of photons and some leptons in the Physical Universe are really constant, i.e., $\tau=0$; that means that they remain into a singularity. That would be a strange situation into the physical Universe since the $t_{C}$ time till today. Otherwise, the General Theory maintains the $c$ limit for photons in the presence of mass; this one, in its turn, generates the curvature of space-time. The curvature decreases as the distance to a given point increases, similarly to the gravitational attraction does. At this point $\left(r_{c}\right)$, the negative gravitational intensity $\Gamma_{G}$ is $10^{-2}$ times lower than the positive expansion intensity $\Gamma_{H}$.

\section{The Thermo-Dynamical Expansion of the Universe}

\subsection{The Photonic Primeval Energy}

The Big Bang has been assumed, from the G. Lemaitre concept, as a "primeval atom". Though it could not exist any type of atom at the Planck temperature, it really would mean a suddenly created energy accumulation, i.e. a photonic source; those photons applied to build the Physical Universe have generated a luminosity in the order of $L_{p u}=10^{59}(\mathrm{erg} / \mathrm{s})$ [9]. Taking into account the assumed duration of the Big Bang of $10^{12}(\mathrm{~s}) \sim 3 \times 10^{4}(\mathrm{y})$, the total energy generated in the Physical Universe formation would have been about $10^{71}$ (erg). However, by including the original energy of CMB photons, a much higher value of the energy produced in the Big Bang could be obtained.

The original energy corresponding to the $\mathrm{CMB}$ can be obtained from the present density of such relic [19], i.e. 416 (photons $/ \mathrm{cm}^{3}$ ). By applying the cosmological principle, the total quantity of $\mathrm{CMB}$ photons in the Universe volume $\left(7.4 \times 10^{85} \mathrm{~cm}^{3}\right)$ should now be $3 \times 10^{82}$ (photons). Since their average temperature is $\sim 2.7(\mathrm{~K})$, it may be estimated an energy of $3.7 \times 10^{-16}$ (erg/photon) which means a total present energy of $1 \times 10^{67}$ (erg) for all CMB photons. From data of reference [20] it may be assumed that the $z$ factor, for a frequency variation since the Big Bang to the $\mathrm{CMB}$, would be $\sim 10^{31}$, (a similar $Z$ value may be deduced from the temperature variation) which means a total energy in the Big Bang period of $2 \times 10^{98}(\mathrm{erg})$, equivalent to a luminosity $L_{B}=2 \times 10^{86}(\mathrm{erg} / \mathrm{s})$. At the Big Bang end there would exist equal number of photons as they are today as CMB 
$\left(3 \times 10^{82}\right)$, plus a small fraction devoted to generate the Physical Universe; so, the average energy of each one should have been $\sim E_{f}=3 \times 10^{15}$ (erg/photon). However, the energy of just one photon whose maximum wavelength would equal the Planck length (as assumed here), it should have been about $1.5 \times 10^{17}$ (erg/photon). That means that the average wavelength of original photons must rather be $\sim 5$ times longer than $L_{p}$, which implies a lowering factor of 5 in the original photons energy to give now $3 \times 10^{16}$ (erg/photon) and a total energy at the Big Bang end of $2 \times 10^{97}$ (erg). So, the luminosity at the Big Bang end would have been $L \sim 2 \times 10^{85}(\mathrm{erg} / \mathrm{s})$. This correction factor 5 in the wavelength of original photons doesn't match with the assumed Planck length. However, there are two possibilities for a coincidence: the first is that the "true" dimension of the Planck length would be 2.5 times bigger than the one here applied; the second possibility is that, accordingly to the quantum theory, a photonic wave is stable in a potential well if it displays an entire multiple of a half wavelength; that is a fact that permits to accept 5 different values of the $(\lambda / 2)$ parameter, so generating a mixture in the original energy spectrum of the Big Bang. Otherwise, the $\mathrm{CMB}$ fluctuations could have been provoked by dispersive interactions of original photons with the subsequent condensed matter.

Besides, the Higgs photon may have energies as high as $\sim 150 \mathrm{GeV}$; that means $\sim 0.25$ (erg/photon). So, it would be feasible that every one of the original photons could generate the necessary number of Higgs photons to start the imminent mass condensation.

The total energy was, apparently, applied to only two purposes: one was the generation of the Physical Universe mass, whose value results $\sim 2 \times 10^{78}$ (erg) if the mass would have a value $\sim 2 \times 10^{57} \mathrm{~g}$ [21]. It means that a minimal fraction $\left(10^{-19}\right)$ of the Big Bang energy was applied to generate the Physical Universe. Accordingly to reference [9] a similar fraction would have been applied to the Physical Universe expansion work. The rest (>99.9\%) was left as the relic of the Big Bang. It would imply that the present electromagnetic spectrum, from radio to gamma and cosmic rays, as well as some leptons, would not come from the Big Bang but from astronomical objects such as the Sun, galaxies and quarks.

\subsection{The Expansion Work of the Physical Universe}

In this expansion, temperature, pressure and volume continuously vary, though entropy does not because there is not any intake or lost of heat in the Universe. The conservation equation for the internal energy of the Universe $(E)$ is, from the 1st Law of Thermodynamics:

$$
E=Q-W(\mathrm{erg})
$$

$Q$ would be the total heat content, and $W$ the work performed by the matter expansion. Initially, at a time lower than Planck's one, the work expansion $W$ may be assumed to be nil and therefore the internal energy $U$ would be equal to the heat content $(E=Q)$. After, in the non-isothermal, non isobaric and adiabatic (though irreversible) case of the Physical Universe, the expansive work will 
be made at expenses of a small fraction of the original internal energy: ( $W=$ $-\delta E)$. So, $E$ is the original internal energy of the Big Bang, which was above calculated as $2 \times 10^{97}(\mathrm{erg}) ; \delta E_{M}$, corresponding to the mass generation, is $2 \times 10^{78}$ (erg), as previously mentioned. By derivation of Equation (19) respect to time and substitution of the density variable, the conservation equation for adiabatic expansion it results:

$$
\dot{\rho}+3 \frac{\dot{a}}{a}\left(\rho+\frac{p}{c^{2}}\right)=0
$$

where $a$ and $\dot{a}$ refer to the scale factor and its time derivative.

However, additional considerations must be made: accordingly to reference [10] "it cannot be pressure forces in a homogeneous universe because such effects can only be generated by a pressure gradient... so, pressure does not provide a force that causes the Universe expansion. Rather, its contribution is entirely through the work done during the expansion". So, it has not sense to search for a pressure value in the Universe expansion process. Otherwise, the state equation for different types of matter has eventually been proposed as:

$$
p=w \rho c^{2}
$$

For vacuum energy, $w=-1$, so assuming that an external negative pressure is the cause of Universe expansion. Such possibility has been discarded by both $\Lambda$ and Hubble models.

There are three ways to determine the work developed in the Physical Universe expansion: to apply the Hubble potential, by means of a classical thermo-dynamical process, and to include a quantum criteria.

1) The Hubble potential acts on the masses present after the decoupling time; so, the to-date work value could be:

$$
W=M_{U} \Gamma_{H} R_{X}(\mathrm{erg})
$$

$M_{u}$ is the mass of the physical Universe and $R_{x}$ is the radial function corresponding to the physical Universe expansion period, $t_{x}=\left(t_{o}-t_{p u}\right)$. Substitution of known values gives a total expansion work of the physical Universe $W_{U} \sim 10 \times$ $10^{76}(\mathrm{erg})$ that was delivered at the spherical shell $R_{x}$, i.e. during the evolution time of the physical Universe, which represents a luminosity of $L=2 \times 10^{59}$ (erg/s), similar to that of reference [9] for the physical Universe. The negative external pressure would be 0 since the empty space density is 0 (different to that of a quantum vacuum). Anyway, the sum of the Physical Universe mass and its expansion work, remain in a fraction lower than $10^{-19}$ of the Big Bang energy.

2) The classic thermo-dynamical expansion work is given by the equation:

$$
W=P V_{u}=n k T\left(\text { dyne } / \mathrm{cm}^{2}\right)
$$

where, $n$ has been calculated as $2.7 \times 10^{82}$ (photons), $k=1.38 \times 10^{-16}$ (erg/K.photon); if $T_{u} \sim 2 \times 10^{10}(\mathrm{~K})$, when the lepton era ends, the product gives $P V=7.5 \times 10^{76}$ (erg). 
3) The quantum method [22] applies the Wien factor $b=7.6 \times 10^{-15}$ $\left(\mathrm{erg} / \mathrm{K} \cdot \mathrm{cm}^{3}\right)$, obtained from statistical physics, to get the thermal pressure; however, since it was derived for an isothermal process, it may not be here applied to an adiabatic expansion. Anyway, the quantum method includes a correction factor for the classical Equation (23) that was here employed, so arriving to a result $W_{U}=6.7 \times 10^{76}(\mathrm{erg})$. The luminosity, in this case, is similar to that corresponding to the evolution of the physical Universe: $L, 1.3 \times 10^{59} \mathrm{erg} / \mathrm{s}$.

\subsection{A Big Bang Design}

Before making additional calculation in the photonic gas, it must be recalled the classical Bose-Einstein statistics for 0 spin particles in a non-isothermal process, i.e.

$$
f(E)=1 /\left(A \mathrm{e}^{E / k T}-1\right)
$$

that is applied to obtain the distribution of photons as a function of temperature [23]; it may not be used here because it represents an equilibrium state at a given temperature $T$, when photons energies vary and most photons accumulate at the lowest energies; it is an ideal though opposed situation to that of the Universe where the photons accumulation happened at the highest temperature. Therefore, it is possible to assume that the only reducing factors of the initial photons energy will be a gravitational (delaying) red-shift and, after the decoupling time, the normal $z$ lowering process driving to the CMB.

In order to prove the initial conditions for the validity of a physical law, it would be important to calculate the expansion velocity of the Big Bang in comparison to that of the Hubble acceleration. So, the general Equation (23) is assumed to represent the adiabatic expansion work of the photonic nucleus; then:

$$
(\dot{R})^{2}=2 n k T / M_{p u}
$$

So, $\dot{R}=5 \times 10^{6}\left(\mathrm{~cm} \cdot \mathrm{s}^{-1}\right)$ would be the final velocity of the Big Bang period, while the velocity obtained from the Hubble acceleration results: $=2.7 \times 10^{5}$ $\left(\mathrm{cm} \cdot \mathrm{s}^{-1}\right)$. This difference must have been much higher at shorter times, which means that the Hubble acceleration law was not significant before the end of the Big Bang period $\left(3 \times 10^{4} \mathrm{y}\right)$. Therefore, this period could be assumed as the one corresponding to inflation. Taking the Planck length $L_{p}=1.6 \times 10^{-33}(\mathrm{~cm})$ as the diameter of an spherical Big Bang, its volume would be $V_{p}=2.15 \times 10^{-99}\left(\mathrm{~cm}^{3}\right)$.

In Table 1 , they are shown the values of expansion velocity $\dot{R}_{H}(\mathrm{~cm} / \mathrm{s})$ and scale factor $R_{H}(\mathrm{~cm})$ of the Space, corresponding to times (s): Planck $\left(t_{p}\right) ; 10^{-10} ; 1$; Big Bang end, $t_{b e} \dot{e}$ decoupling time $t_{\dot{d}}$ Physical Universe formation time $t_{p u \dot{ }} c$ time, $t_{\dot{c}}$ and present times: $t_{11}$ for the Physical Universe and $t_{o}$ for Space. Four values for luminosity $L_{,}(\mathrm{erg} / \mathrm{s})$ are also shown for times $t_{b e}, t_{d}$ and $t_{o}$ times, as well as to the period $\left(t_{c}-t_{b e}\right)$. The present scale factor of the Physical Universe is assumed as the observable Universe: the distance to the most distant object (GN-Z-11), $R_{11} \sim 2 \times 10^{27}(\mathrm{~cm})$. 
Table 1. Expansion velocity $(V)$, and scale factor $(R)$ of the Space, according to the Planck parameters (first line) and to the Hubble acceleration (next 8 lines). The luminosity $L$ (erg/s) of Space is shown in the fourth column for: the Big Bang end $\left(t_{b e}\right)$, the decoupling time $\left(t_{d}\right)$, the $\left(t_{c}\right)$ time and the present time $\left(t_{o}\right)$.

\begin{tabular}{cccc}
\hline$t(\mathrm{~s})$ & $\dot{R}(\mathrm{~cm} / \mathrm{s})$ & $R(\mathrm{~cm})$ & \multicolumn{1}{c}{$(\mathrm{erg} / \mathrm{s})$} \\
\hline$t_{p}=5.4 \times 10^{-44}$ & $c$ & $L_{p}=1.6 \times 10^{-33}$ & \\
$t=10^{-10}$ & $2.7 \times 10^{-17}$ & $R_{-10}=1.4 \times 10^{27}$ & \\
$t=1$ & $2.7 \times 10^{-7}$ & $R_{1}=1.4 \times 10^{-7}$ & \\
$t_{b e}=10^{12}$ & $2.7 \times 10^{5}$ & $R_{b e}=1.5 \times 10^{17}$ & $L_{b e}=2 \times 10^{85}$ \\
$t_{d}=1.5 \times 10^{13}$ & $3.7 \times 10^{6}$ & $R_{d}=4.6 \times 10^{19}$ & $L_{d}=6 \times 10^{83}$ \\
$t_{p u}=3.1 \times 10^{16}$ & $0.3 c$ & $R_{p u}=3.0 \times 10^{24}$ & \\
$t_{c}=1.1 \times 10^{17}$ & $c$ & $R_{c}=1.6 \times 10^{27}$ & $L_{j}\left(t_{c}-t_{b e}\right)=4.5 \times 10^{80}$ \\
$t_{l 1}=t_{o}-t_{p u}$ & $3 c$ & $R_{11}=2.0 \times 10^{27}$ & \\
$t_{o}=4.4 \times 10^{17}$ & $4 c$ & $R_{o}=2.6 \times 10^{28}$ & $L_{o}=5 \times 10^{79}$ \\
\hline
\end{tabular}

\section{The Photonic Equation of Continuity}

The operator of the convective derivative has been usually applied to density and time variables such as velocity, momentum and kinetic energy. The same operator may also be applied to these parameters when they are not expressed as function of masses but of frequencies, such in the photons case. Applying the convective operator to the photon energy, $E=h v$, if $E(r, t)$, it gives:

$$
D h v / d t=\partial h v / \partial t+c \partial h v / \partial r
$$

Substitution of $c$ in $\partial r$ drives to the equation of continuity:

$$
\frac{D E}{d t}=2 h\left(\frac{\partial v}{\partial t}\right)
$$

This rather unexpected result may be applied to an example with the above mentioned parameters if $h \partial v$ would be assumed as $\Delta E=\left(E_{c}-E_{b e}\right)$ and $\partial t$ as $\Delta t=\left(t_{c}-t_{b e}\right)$ to give:

$$
\frac{D E}{d t}=2\left[\frac{\Delta E}{\Delta t}\right](\mathrm{erg} / \mathrm{s})
$$

where, the sub-fix ${ }_{\text {be }}$ refers to the Big Bang end time $\left(t_{b e}\right)$ and ${ }_{c}$ to the $t_{c}$ time. Equation (22) gives a result $D E / d t=4.5 \times 10^{80}(\mathrm{erg} / \mathrm{s})$; it would be the rate of outgoing energy, since the Big Bang end time till the $t_{c}$ time. This is the luminosity $\left(L_{c}\right)$ of the Universe corresponding to the period $\left(t_{c}-t_{b e}\right)$. The Jacobian for this energy in adiabatic expansion may be expressed as:

$$
J(E, S)=\frac{J(E, S)}{J(t, S)}=\left(\frac{\partial E}{\partial t}\right)_{s}
$$

Substitution gives a similar result:

$$
(D E / d t)_{s}=4.3 \times 10^{80}(\mathrm{erg})
$$




\section{Conclusions}

1) It has been assumed that, at the Big Bang, there were created four realities: time, space, matter and physical laws. Several references [24], [25], [26] ask about the time creation of the physical laws. If the above mentioned constancy of the Space acceleration $\Gamma_{H}$ is correct, it would represent the first physical law created at the Big Bang, respect to Space, together with the gravitational law $\Gamma_{G}$, respect to matter; however, $\Gamma_{H}$ would appear evident only after the Big Bang end, as well as $c$ would be freely manifest till the $t_{c}$ time. Additional parameters, such as Luminosity, are summarized in Table 1. It is also assumed that the Universe is the total accelerating Space that contains the Physical Universe and an external vacuum Space.

2) The Big Bang was not an instantaneous event; it remained for $10^{12}(\mathrm{~s})$ or $3 \times$ $10^{4}$ (y) [9]. Given both the length and the expansion velocity at this stage, it may be assumed to correspond to the Inflation period.

3) The $c$ time $\left(t_{c}\right)$ was defined as that when Space and the Physical Universe reached together the $c$ value of expansion velocity; at such time, both Space and Physical Universe had a radius $\boldsymbol{r}_{\boldsymbol{c}}$ In spite the gravitational law had continuously been opposed to the Physical Universe expansion, the Space expansion has independently occurred due to the constancy of the Hubble acceleration expressed by $\Gamma_{H}$. However, inside the Physical Universe, matter velocity has kept a maximum velocity $c$ as a universal constant, a consequence of the space-time curvature. So, the furthest tangential velocity of the Physical Universe, at the $t_{c}$ time, could also have been $\mathcal{c}$, an assumed data that had permitted to calculate the Physical Universe angular momentum as $\mathcal{L}=4.8 \times 10^{94}$ (erg.s).

4) According to Special Relativity, at c velocity the proper time becomes nil, i.e. $\tau=0$, which implies a time freezing, as well that matter felt in a singularity; it would be difficult to define the meaning and length of such singularity. Also, it has been mentioned [18] the theoretical possibility of an imaginary time $\left(\tau_{i}\right)$ if the limits of the Physical Universe overpasses the $c$ velocity. It has been shown in a simplified Euclidic Space ${ }^{3}$ [26].

5) The to-date called Hubble Length, $L_{H}=c / H$, had been defined as the distance where the space expansion rate $\dot{R}$ is just $c$, so, farther $L_{H}, \dot{R}>c$. However, $H$ is usually assumed as constant though it should rather be determined as $H_{o}$, i.e. a function of the present scale factor $R_{o}$, obtained from the constant value of $\boldsymbol{\Gamma}_{H}$. Besides, the $H_{o}$ value deduced in reference [1] has been modified in this paper to $H_{o}=3.2 \times 10^{-18}\left(\mathrm{~s}^{-1}\right)$; the value of $\boldsymbol{\Gamma}_{H}$ is $2.65 \times 10^{-7}\left(\mathrm{~cm} \cdot \mathrm{s}^{-2}\right)$.

6) The parameter $\Omega$ has no sense at the expanding limit of the Physical Universe since the density of the external space is 0 . As well, the $q$ parameter must be $>0$ in an expanding Physical Universe, because every one of its factors is $>0$. Respect to the Hubble parameter, it was here deduced the equation $H(t)=\sqrt{2} / t \quad$ (8) for an expansive Universe.

${ }^{3}$ The Euclidic name has been proposed by S. Hawkings for a Euclidean Space that includes an imaginary time coordinate [18]. 
7) The expansive work calculation was intended by 3 ways: the Hubble potential, the thermo-dynamical process and a brief quantum concept. They gave $W \sim$ $7.4 \times 10^{76}$ (erg).

8) The Big Bang was a source of photons, staying for $3 \times 10^{4}$ years to liberate a total energy of $2 \times 10^{97}$ (ergs) at a constant true velocity $c$ that seemed impossible into the Big Bang tangle. A minimum part of this energy $\left(\sim 10^{-21}\right)$ was applied to build and expand the Physical Universe; the rest of photons decayed necessarily till the present known CMB energy, without any foreseeable task.

9) The convective operator was applied to photons energy, as a function of frequency, to get an Equation (27) derived from the assumption that energy is a function of time and distance. As well, the Jacobian obtained for the adiabatic expansion gave a similar result for Luminosity.

10) The above calculated initial energy of photons is high enough to generate the necessary number of Higgs photons in order to produce mass condensation. The above mentioned totality of initial photons would not be mono-energetic; their variation is tied, between several factors, to the Heisenberg indetermination of the Planck length, which results $\sim 5$ times the proper length. Fluctuations of $\mathrm{CMB}$ photons could be originated by dispersive interactions with the Physical Universe matter.

11) Some conclusions require the experimental determination of the true Space acceleration expansion, here deduced as $\Gamma_{H}=2.65 \times 10^{-7}\left(\mathrm{~cm} \cdot \mathrm{s}^{-2}\right)$.

\section{Acknowledgements}

The author thanks to M. A. Zúñiga for his kind collaboration in this work.

\section{Conflicts of Interest}

The authors declare no conflicts of interest regarding the publication of this paper.

\section{References}

[1] Lartigue, J.G. (2016) Journal of Modern Physics, 7, 1607-1615. https://doi.org/10.4236/jmp.2016.712145

[2] Mather, J.C. (2006) From the Big Bang to the Nobel Prize. Lecture.

[3] Ford, I. (2013) Statistical Physics. J. Wiley, New York, 187-199. https://doi.org/10.1002/9781118597507

[4] Pacavic, A. (1963) Thérmodynamique Statistique. Masson et Cie, Paris, 238.

[5] Chen, J. (2010) The Relativistic Dynamics of a Photon Gas. p. 6.

[6] Bergstrom, L. and Goobar, A. (2004) Cosmology and Particle Astrophysics. 2nd Edition, Springer, Berlin, 60.

[7] Lartigue, J.G. (2017) Journal of Modern Physics, 8, 6, Eq. (4.9).

[8] Salvo, R., et al. (2017) Science, 358, 765-768. https://doi.org/10.1126/science.aan4054

[9] Hogan, C.J. (1999) Energy Flow in the Universe. <arXiv:astro-ph/9912110v|>. 
[10] Lidsey, J.E. (2014) Cosmology Course ASTM108/PHY7010U, eq. (4.2), Queen Mary School, London.

[11] Lartigue, J.G. (2016) Journal of Modern Physics, 7, 1607-1615, eq. (3.3). https://doi.org/10.4236/jmp.2016.712145

[12] Sartory, L. (1996) Understanding Relativity. University of California, Los Angeles, 313.

[13] Lidsey, J. op. cit. eq. (2.28).

[14] Sartory, L.(1996), op. cit. , p. 325, Figure 9-12.

[15] Gleiser, M. (2013) The Origin of the Universe. https://www.npr.org/section/13.7

[16] Tolman, R. (1958) Relativity, Thermodynamics and Cosmology. Oxford Press, Oxford, 487 .

[17] Einstein, A., et al. (1923) The Principle of Relativity. Dover Pub., 106.

[18] Hawkings, S. (1988) Historia del tiempo. Editorial Crítica, México, 179.

[19] Leff, H.S. (2002) American Journal of Physics, 70, 792-797. https://doi.org/10.1119/1.1479743

[20] Sartory, L. (1996), op. cit. p. 342, Table 9.1.

[21] Johnson, J.R. (2013) Comprehending the Cosmos. 2nd Edition, Create Space Independent Publication, 204, 264.

[22] Leff, H. S. (2002) op. cit. p. 793.

[23] Krane, K. (1983) Modern Physics. Wiley, New York.

[24] Davies, P. (1995) L’Esprit de Dieu. Éditions du Rocher, Paris, 83.

[25] Hawkings, S. (op. cit.) p. 164.

[26] Lartigue, J.G. (2018) The Universe Origin and Destiny. Journal of Modern Physics, 9, 1823, Figure 3. 